\title{
Mesoscopic Ordering Effects in Single-Crystalline Thin Films of Lanthanum Manganites
}

\author{
Z.A. Samoilenko ${ }^{a}$, V.D. OkuneV ${ }^{a}$, E.I. Pushenko ${ }^{a}$, \\ A. Abal'osheV ${ }^{b}$, M. Baran ${ }^{b}$, P. Gierlowski ${ }^{b}$, \\ S.J. LEWANDOWSKI ${ }^{b}$ AND R. SZYMCZAK ${ }^{b}$ \\ ${ }^{a}$ Donetsk Institute for Physics \& Technology \\ National Academy of Sciences of Ukraine (Donetsk) \\ ${ }^{b}$ Institute of Physics, Polish Academy of Sciences \\ al. Lotników 32/46, 02-668 Warszawa, Poland
}

\begin{abstract}
We have investigated thin films of lanthanum manganites epitaxially grown by pulsed laser deposition on single-crystalline substrates. X-ray diffraction studies show that the characteristic feature of the investigated films is a superposition of fractions with long range atomic order in the basic single-crystalline matrix, and mesoscopic order in the clusterized structure encompassing the $\mathrm{Mn}-\mathrm{O}$ layers. A detailed analysis of diffusive scattering shows that the size of metallic clusters and their volume concentration in the dielectric matrix varies. In the case of a small size and concentration, the metallic clusters in low temperature behave as quantum dots and their presence leads to a tunneling mechanism of conductivity with characteristic $R(T)=$ const. In the case of large cluster size, the discrete energy levels become smeared. It appears also that the optical, transport, and magnetic properties of the investigated films depend critically on the distribution of $\mathrm{Mn}^{2+}, \mathrm{Mn}^{3+}$, and $\mathrm{Mn}^{4+}$ ions in the clusters.
\end{abstract}

PACS numbers: $75.30 . \mathrm{Kz}, 75.47 . \mathrm{Lx}, 61.50 . \mathrm{Ks}$

\section{Introduction}

Physical properties of the family of $\mathrm{LaCa}(\mathrm{Sr}) \mathrm{MnO}_{3}$ perovskite manganites are determined by interactions of their electron, atomic, and magnetic subsystems. 
The behavior of these compounds, including their colossal magnetoresistance, depends on their atomic order and the presence of compositional or structural inhomogeneities $[1,2]$. In epitaxial thin films exhibiting a high quality crystalline structure the principal role in their electrical and optical properties plays the presence of clusters with the metallic conductivity, which are embedded in the insulator host matrix [3]. The electrical resistivity of the films is limited by tunneling of electrons between clusters. The concentration and types of such clusters strongly depend on the growth conditions of the films and are determined by the competition of orthorhombic and rhombohedral structures [3].

In this work we have investigated the local cluster structure and its influence on the electric and magnetic properties of $\mathrm{LaCa}(\mathrm{Sr}) \mathrm{MnO}_{3}$ thin films obtained by pulsed laser (KrF) deposition on different monocrystalline substrates.

\section{Results and discussion}

The local cluster structure can be determined by X-ray coherent diffusive scattering. This scattering was analyzed in order to determine the peculiarities of cluster structure of the films deposited using various ambient conditions.

Typical debyegrams obtained in $\mathrm{Cr}$ radiation for $\mathrm{La}_{2 / 3} \mathrm{Ca}_{1 / 3} \mathrm{MnO}_{3}$ and $\mathrm{La}_{0.6} \mathrm{Sr}_{0.2} \mathrm{Mn}_{1.2} \mathrm{O}_{3}$ manganites have the Laue form. The most intense Laue reflex apparently is the result of X-ray interference on the crystallographic planes representing the dominant atomic ordering of the film. Its angular $\theta$ position characterizes the $(d, h k l)$ crystallographic parameters and the phase adherence of the relevant planes. The $\theta$ values of the other reflexes positioned on the axial line of the debyegram follow the Wulff-Bragg law and allow the determination of the ( $h k l)$ indexes of the corresponding planes, participating in the diffraction. Micro photometric measurements along this line render possible a quantitative comparison of the intensity of X-ray scattering in a given $\theta$-interval for samples with different atomic order in the corresponding structural groups of planes, even for identical nominal chemical composition, as shown e.g. in Fig. 1.

From this point of view, the most interesting are structural groups formed during the growth of manganese-oxygen complexes of the perovskite sublattice. It is known $[1,2]$ that these complexes exhibit a wide variety of lengths and angles of $\mathrm{Mn}-\mathrm{O}$ bonds, as well as a selection of charge states: $\mathrm{Mn}^{2+}, \mathrm{Mn}^{3+}$, and $\mathrm{Mn}^{4+}$. Basing on the published data, we have determined an interval $(31-36)^{\circ}$ of the diffraction angles, capturing these reflexes, which take on the diffusive form because of the above listed variations in the scattering centers of the analyzed local structure. Varying the film growth parameters induces substantial changes in the matrix and in the cluster structure. The fact that cluster boundaries are smeared facilitates local changes of atomic order. The detailed analysis of diffusive scattering shows that the size of metallic clusters in the dielectric matrix varies from $40-70 \AA$ to $140-200 \AA$, and their volume concentration - from $1-10 \%$ to $20-50 \%$. 

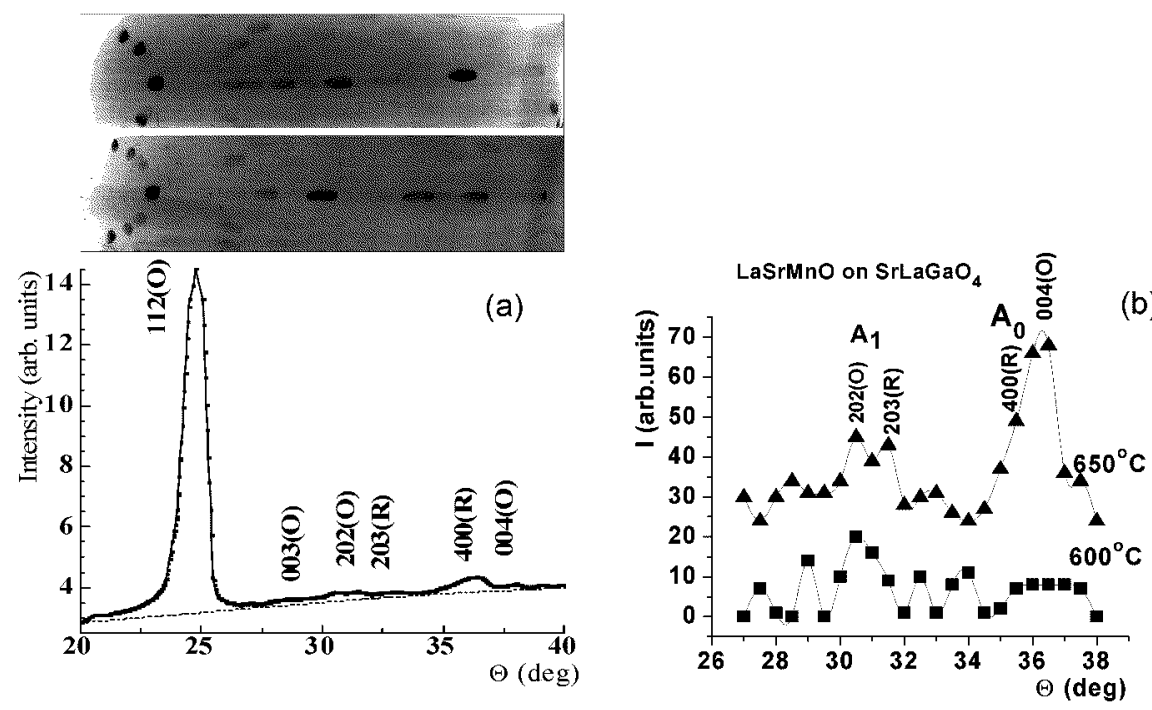

Fig. 1. Typical diffractograms of manganite films (a). Diffusive maxima are characteristic of clusterized regions of the crystalline structure, here shown in detail for films undergoing the $\mathrm{R} \rightarrow \mathrm{O}$ transition (b).

In the first case (a small size and concentration), the metallic clusters in low temperature behave as quantum dots and their presence leads to a tunneling mechanism of conductivity with characteristic $R(T)=$ const dependence at low enough temperatures. In the second case (a large cluster size), the discrete energy levels become smeared $(\Delta \ll k T)$ and the electrical properties of the samples become independent of quantum size effects.

\section{Results and discussion}

We describe further three groups of experiments, in which we analyzed the effect of changes in the local structure of clusters on electronic and magnetic properties of $\operatorname{LaSr}(\mathrm{Ca}) \mathrm{MnO}$ films.

1. We have observed qualitative changes in the shape of angular diffusive scattering spectra accompanying the phase transition from rhombohedral (R) to orthorhombic (O) film structure, occurring as a result of varying the film growth temperature in the $600^{\circ} \mathrm{C}<T_{\mathrm{s}}<670^{\circ} \mathrm{C}$ range (see Fig. 1). For $T_{\mathrm{s}}=600^{\circ} \mathrm{C}$, characteristic are structures with dominating atomic order in the form of distant (Mn-O) planes of (202)-O and (203)-R types with weakly ionized $\mathrm{Mn}^{2+}$ and $\mathrm{Mn}^{(2 \div 3)+}$ ions (structural group $A_{1}$ ). Higher growth temperatures lead to the formation of closely spaced planes of (400)-R and (004)-O types containing strongly ionized $\mathrm{Mn}^{3+}$ and $\mathrm{Mn}^{(3 \div 4)+}$ ions (structural group $A_{0}$ ). These findings are based on the assumptions that the interplanar distance $d \sim 1 / \sin \Theta$, and 
that with increased ionization the distance between interacting ions is reduced. Structural changes $\mathrm{R} \rightarrow \mathrm{O}$ are accompanied by qualitative changes in the temperature dependence of film resistivity. For R-phase this dependence is exhibiting the $R(T)=$ const feature, while for O-phase $R(T)$ accepts values from the $\left[R_{\max }, R_{\min }\right]$ interval, as seen in Fig. 2.
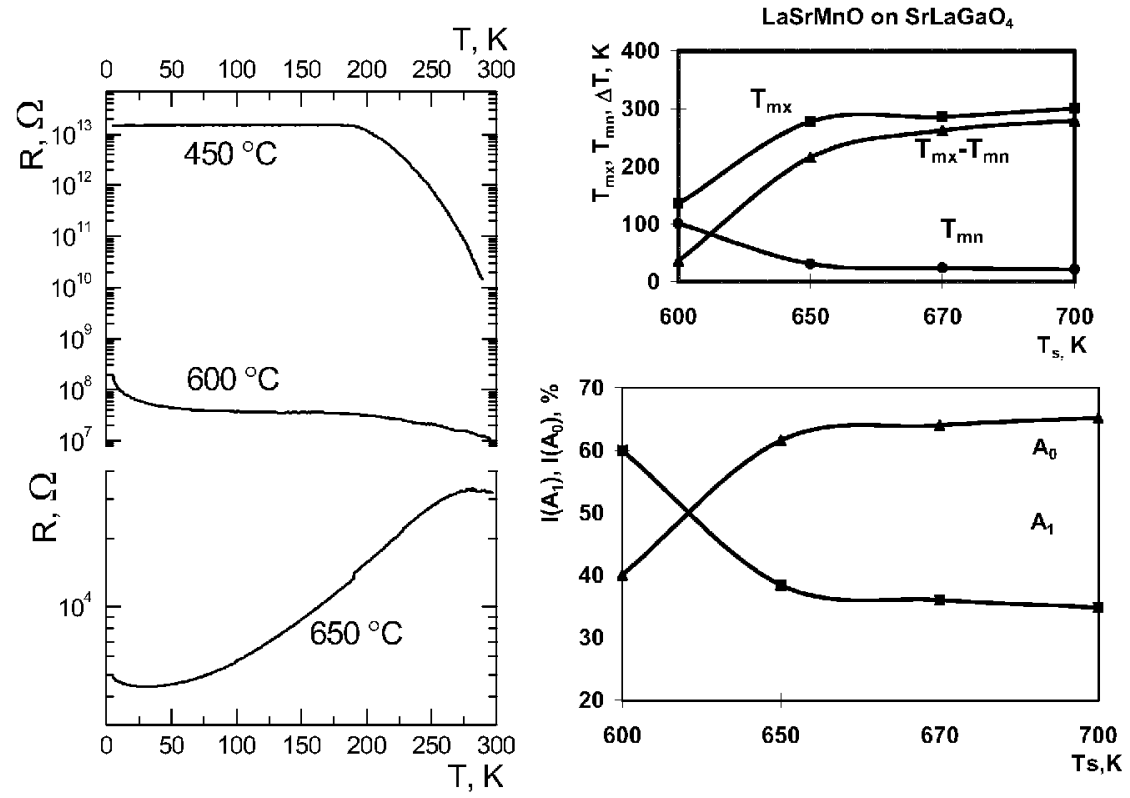

Fig. 2. Changes induced by $\mathrm{R} \rightarrow \mathrm{O}$ transition in characteristic features of temperature dependence of film resistivity, from $R(T)=$ const to $R(T)=\left[R_{\max }, R_{\min }\right]$, and the dependence of "metallic" interval of $R(T)$ on the cluster concentration in $A_{1}$ and $A_{0}$ groups.

These changes in the $R(T)$ curves reflect fundamental changes in the mechanisms of charge tunneling between metallic clusters: in the case of $R$-phase, at low enough temperatures, the clusters behave as quantum dots with discrete energy levels, while for O-phase, at high enough concentration of metallic clusters, the energy levels become quasi-continuous because of a high density of states.

2. An increase in growth temperature $T_{\mathrm{s}}$ of $\mathrm{LaSrMnO}$ films from $600^{\circ} \mathrm{C}$ to $700^{\circ} \mathrm{C}$ leads to an increase (eventually saturated) in relative concentration of $A_{0}$ clusters, represented by (400)- $\mathrm{R}$ and (004)-O planes (see $I\left(A_{0}\right)$ curves in Fig. 2), accompanied simultaneously by a symmetrical decrease in the concentration of clusters, which belong to the $A_{1}$ group (see $I\left(A_{1}\right)$ curves in Fig. 2). These changes in the local ordering of clusterized regions of the crystalline structure are reflected in the lengthened "metallic" interval on the $R(T) \in\left[R_{\max }, R_{\min }\right]$ characteristic: $T_{\max }$ increases, while $T_{\min }$ decreases, as shown in the relevant characteristics. 
Lengthening of the $\Delta T=T_{\max }-T_{\min }$ interval denotes strengthened interaction between metallic clusters in the dielectric crystalline matrix [3], which is caused by a decreased cluster separation due to the increased concentration of the metallic phase.

3. By varying the film growth temperature and using various substrate materials, we obtained $\mathrm{LaSr}(\mathrm{Ca}) \mathrm{MnO}$ films differing by the details of atomic ordering in the clusterized regions, as indicated by the intensity changes of X-ray scattering in discrete angular directions, shown by the $I_{h k l}$ characteristics in Fig. 3 . These structural differences apparently magnified the differences in magnetic properties of our films. In particular, the saturation magnetization of individual samples could differ 14 times.

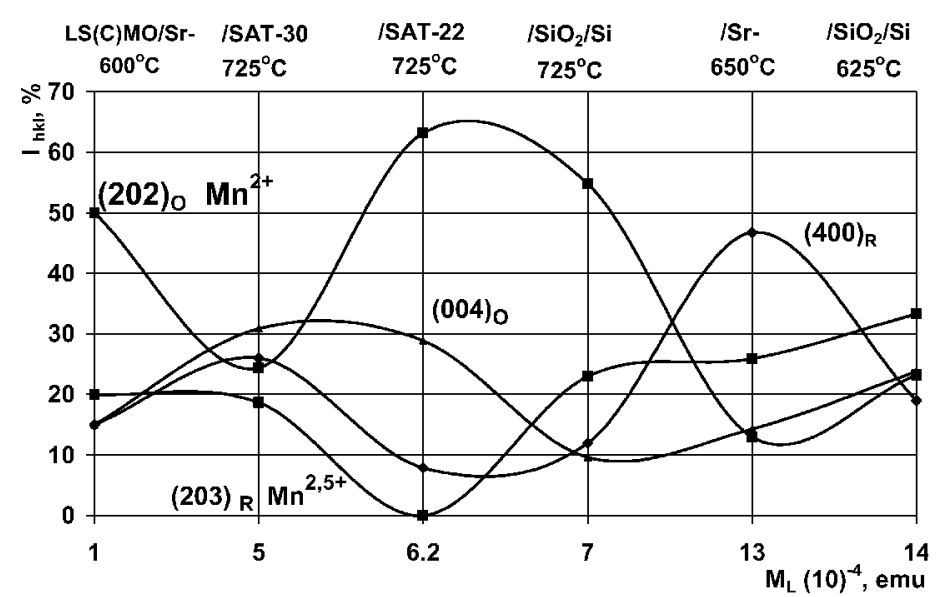

Fig. 3. X-ray scattering intensity $I_{h k l}$ at discrete $\theta$ values for different manganite films.

Let us draw our attention to two groups of samples, paired by identical chemical composition and identical substrates, but differing in growth temperature $T_{\mathrm{s}}$. We can compare the X-ray scattering intensities $I_{h k l}$ in discrete angles $\theta$, assuming that by increasing $\theta$ and moving from structural $\mathrm{Mn}-\mathrm{O}$ groups with higher interplane distances $d$ towards groups with lower $d$ values, i.e. by moving along the path $(202)_{\mathrm{O}} \rightarrow(203)_{\mathrm{R}} \rightarrow(400)_{\mathrm{R}} \rightarrow(004)_{\mathrm{O}}$, we increase also the manganese ionization and follow the path $\mathrm{Mn}^{2+} \rightarrow \mathrm{Mn}^{2.5+} \rightarrow \mathrm{Mn}^{3.5+} \rightarrow \mathrm{Mn}^{4+} . \mathrm{La}_{0.6} \mathrm{Sr}_{0.2} \mathrm{Mn}_{1.2} \mathrm{O}_{3}$ films grown on $\mathrm{SrLaGaO}_{4}$ at $T_{\mathrm{s}}=650^{\circ} \mathrm{C}$ exhibit saturation magnetization 14 times greater than that of the same films grown at $T_{\mathrm{s}}=650^{\circ} \mathrm{C}$ (Fig. 4). The structural investigation of these samples (see also Fig. 4) reveals that the higher magnetization of $T_{\mathrm{s}}=650^{\circ} \mathrm{C}$ sample is related to a higher ( 3 times) concentration of clusters with closely spaced planes containing highly ionized manganese $\mathrm{Mn}^{3+}$.

The manganite films grown on oxidized $\mathrm{Si}$ substrates at $T_{\mathrm{s}}=625^{\circ} \mathrm{C}$ show higher $\left(2.8\right.$ times) saturation magnetization than those grown at $T_{\mathrm{s}}=725^{\circ} \mathrm{C}$ (see 

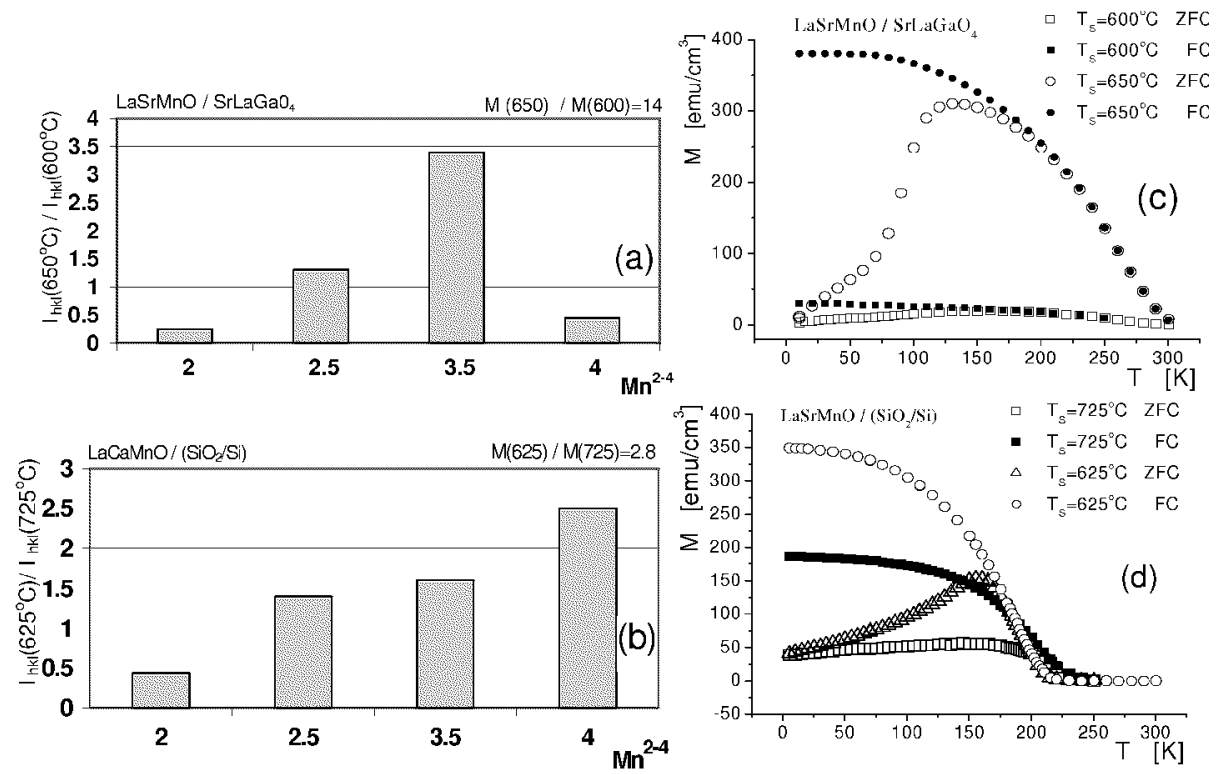

Fig. 4. Graphical representation of $I_{h k l}$ ratios for clusterized regions of the compared structures $(\mathrm{a}, \mathrm{b})$ and saturation magnetization measured in $H=100$ Oe in a function of temperature (c,d) for $\mathrm{LaSr}(\mathrm{Ca}) \mathrm{MnO}$ films grown at $T_{\mathrm{s}}=600 \div 725^{\circ} \mathrm{C}$.

Fig. 4). The structural analysis reveals, however, that the higher magnetization of $T_{\mathrm{s}}=625^{\circ} \mathrm{C}$ sample is also related to higher $\left(1.5\right.$ times) concentration of $\mathrm{Mn}^{3.5+}$ ions (Fig. 4). In this case, magnetic ordering is reduced because of increased antiferromagnetic interactions introduced by the accompanying high concentration of clusters with $\mathrm{Mn}^{4+}$ ions [4].

To conclude, our results clearly demonstrate the explicit effect of local crystallographic structure of $\mathrm{Mn}-\mathrm{O}$ clusters on the physical properties of manganites.

\section{Acknowledgment}

This work was supported by the State Committee for Scientific Research grant PBZ-KBN-013/T08/19. The authors would like to acknowledge valuable discussions with Prof. M. Kopcewicz.

\section{References}

[1] J.M.D. Coey, M. Viret, S. von Molnar, Adv. Phys. 48, 167 (1999).

[2] Q. Huang, A. Santoro, J.W. Lynn, R.W. Erwin, J.A. Borchers, J.L. Peng, R.L. Greene. Phys. Rev. B 55, 14987 (1997).

[3] Z.A. Samoilenko, V.D. Okunev, E.I. Pushenko, T.A. D'yachenko, A. Cherenkov, P. Gierlowski, S.J. Lewandowski, A. Abal'oshev, A. Klimov, A. Szewczyk. Techn. Phys, 48, 250 (2003).

[4] E.L. Nagaev, Usp. Fiz. Nauk 166, 833 (1996). 